\title{
Correlation Between CT-Simulator and Dynamic Magnetic Resonance Images in Determining The Location of Gross Tumor Volume in Radiotherapy Treatment Planning for Lung Cancer
}

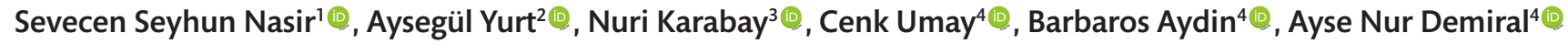 \\ 1Dr. Lutfi Kırdar Kartal Training and Research State Hospital, Radiation Oncology, İstanbul, Turkey \\ ${ }^{2}$ Dokuz Eylül University, Medical Physics, İzmir, Turkey \\ ${ }^{3}$ Dokuz Eylül University, Radiology, İzmir, Turkey \\ ${ }^{4}$ Dokuz Eylül University, Radiation Oncology, Izmir, Turkey
}

Address for Correspondence: Barbaros Aydin, E-mail: barbaros aydin@hotmal.com

Received: 13.05.2018; Accepted: 02.11.2018; Available Online Date: 23.01.2019

(C) Copyright 2018 by Dokuz Eylül University, Institute of Health Sciences - Available online at www.jbachs.org

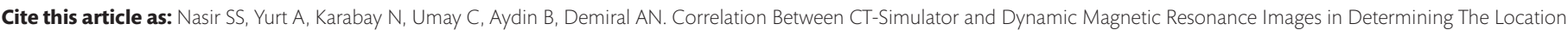
of Gross Tumor Volume in Radiotherapy Treatment Planning for Lung Cancer.J Basic Clin Health Sci 2019; 3:22-29. https://doi.org/10.30621/jbachs.2019.517

\begin{abstract}
Objective: This study aimed to determine the accuracy of the average tumor location in CT-simulator images and PTV margins in radiotherapy treatment planning by detecting lung tumor mobility in dynamic MRI scans of patients presenting with lung cancer.

Materials and Method: Dynamic MRI examination was performed in 12 lung cancer patients scheduled for 3-dimensional conformal RT (3D-CRT) using CT-simulator. In this study, maximum tumor displacement (CC-MRI, ML-MRI, AP-MRI) was measured that occurred in CC, $\mathrm{AP}$ and $\mathrm{ML}$ axes in expiratory and inspiratory phases of dynamic MRI. The distance from the upper limit of GTV to the reference line (CC-CT) was measured in CT-simulator images. CC-CT and CCmean-MRI values were compared with the Wilcoxon signed-rank test. Furthermore, correlation of these values was evaluated with Spearman's correlation test.

Results: In comparison of CC-CT and CCmean-MRI values, no statistically significant difference was detected between CT-simulator and dynamic MRI modalities ( $p=0.172$ ). In addition, a high level of correlation was found between these two imaging methods $(p<0.0001)$.

Conclusion: In our study, the maximum difference between the mean and the CC distances from the upper limit of GTV to the reference line at inspiration and expiration phases in dynamic MR images was $0.4 \mathrm{~cm}$. In general, it can be concluded that the tumor location detected in CTsimulator and dynamic MRI correlate well. However, tumor mobility during respiration is high particularly for small and/or, peripheral and/or lower lobe tumors; thus for such cases it is recommended to get use of dynamic MRI in radiotherapy treatment planning.

Key words: lung cancer, respiratory motion, radiotherapy, dynamic magnetic resonance imaging, CT-simulator
\end{abstract}

\section{INTRODUCTION}

Various imaging techniques are available for staging lung cancer (LC). Computerized Tomography (CT) provides detailed anatomical information by using cross-sectional imaging, and also can generate three-dimensional images in different planes. Magnetic resonance imaging (MRI) is an imaging method which does not involve ionizing radiation and which is capable of performing imaging in any plane. However, it is used as a secondary imaging method in the assessment of LC (1). MRI is capable of characterizing solid pulmonary lesions by using the same morphological criteria described for CT (2). In addition, $M R I$ is superior to $C T$ in differentiating the lymph nodes from vasculature. Therefore, MRI provides more accurate results especially in the assessment of hilar and aorticopulmonary lymph nodes (3).
For three-dimensional conformal radiotherapy (3DCRT) firstly cross-sectional images of the treatment area are acquired in CTsimulator using immobilization devices. Then, these images are transferred to a computerized treatment planning system (TPS). In TPS, the contours of the patient's anatomical structures and tumor are drawn on transverse cross sections of the images and also a variety of target volumes containing the tumor region are formed by the radiation oncologist (4).

Depending on the respiration, tumor and organs may display significant motion, and the size of the motion may vary depending on the patient's anatomical and physiological status as well as size and location of the tumor. During normal respiration, CC 
motion up to $3 \mathrm{~cm}$ can occur in lung tumors, which can lead to significant differences between planned and actually delivered dose distributions.

CT-simulator images used in 3DCRT planning of LC may not represent the actual location of the tumor because they are acquired in a shorter time than the total time of all phases of respiration. In the past, various methods have been used to monitor tumor motion in order to identify PTV more accurately $(5,6)$. Fluoroscopy, active breathing control $(A B C)$, "gating" with markers placed on the skin, portal imaging of implanted markers inserted inside the tumor and CT imaging, and MR-guided radiotherapy have been used in monitoring of tumor motion. In addition to these methods, dynamic images of respiratory motion are obtained using MRI with high spatial and temporal resolution recently $(7,8)$. In this examination, which is named as dynamic MRI, the amount and direction of tumor motion can be detected more accurately with imaging throughout all phases of respiration.

Accordingly, in this study, it was aimed to compare and correlate craniocaudal (CC) tumor location on CT-simulator images and average CC tumor location on Dynamic MRI images acquired for RT planning during the respiratory cycle as well as to evaluate maximum tumor motion in CC, AP and ML axes during inspiration and expiration with dynamic MRI.

\section{MATERIALS AND METHODS}

This study was conducted under the approval by the Institutional Review Board. In our study, 12 patients with LC whose 3D-CRT plans were created using a CT-simulator and TPS in Radiation Oncology Department were selected. The study inclusion criteria were age being $\geq 18$ and $\leq 85$, presence of histological diagnosis of primary or metastatic LC, having an indication for neoadjuvant, definitive or palliative radiotherapy (RT), presence of an identifiable tumor GTV on CT-simulator and dynamic MR images. This study was conducted in accordance with ethical standards under the responsibility of the local committee which evaluates the clinical studies.

\section{MRI and CT}

CT images were taken while the patients were in the supine position on the lung "board" with arms folded above the head and allowing free breathing (FB). Images were acquired in the CC direction using "Siemens" brand "Somatom" model CT-simulator, at $130 \mathrm{kV}, 90 \mathrm{mAs}$, with $5 \mathrm{~mm}$ of cross section interval, and $5 \mathrm{~mm}$ of increments (table movement).

Images were obtained with 1.5 tesla MRI scanner (Philips, Achieva model), using body coil ( $\mathrm{Q}$ body). The patients were placed in the $\mathrm{Q}$ body coil in the same position as in CT. Dynamic MR images were obtained by using balanced Turbo Field Echo (DYN-sBTFE) sequences and imaging parameters were selected as TR/TE: 3.6/1.69 ms, Field of View (FOV): 380 mm, Flip Angle: 70, Matrix: $192 \times 256$, slice thickness $5 \mathrm{~mm}$. In dynamic examination, 15 crosssections were taken for each lesion at 3 different levels passing through the lesion, in the axial, coronal, and sagittal planes, and the total scanning time was determined as 31 seconds. During acquisition of MRI images, breath-holding or respiratory gating was not implemented in order to simulate the situation during $\mathrm{RT}$; instead, images were obtained during spontaneous breathing.

Cross sectional images taken in the CT-simulator were transferred to Oncentra Masterplan TPS. GTVs that had been drawn by the radiation oncologists on cross-sectional images of patients undergoing 3DCRT, were controlled and corrected by a radiology specialist.

Determination of average tumor location and measurement of maximum tumor mobility

Selection of the anatomic reference point used in this study was performed similarly as in the study of Plathow and colleagues (9).

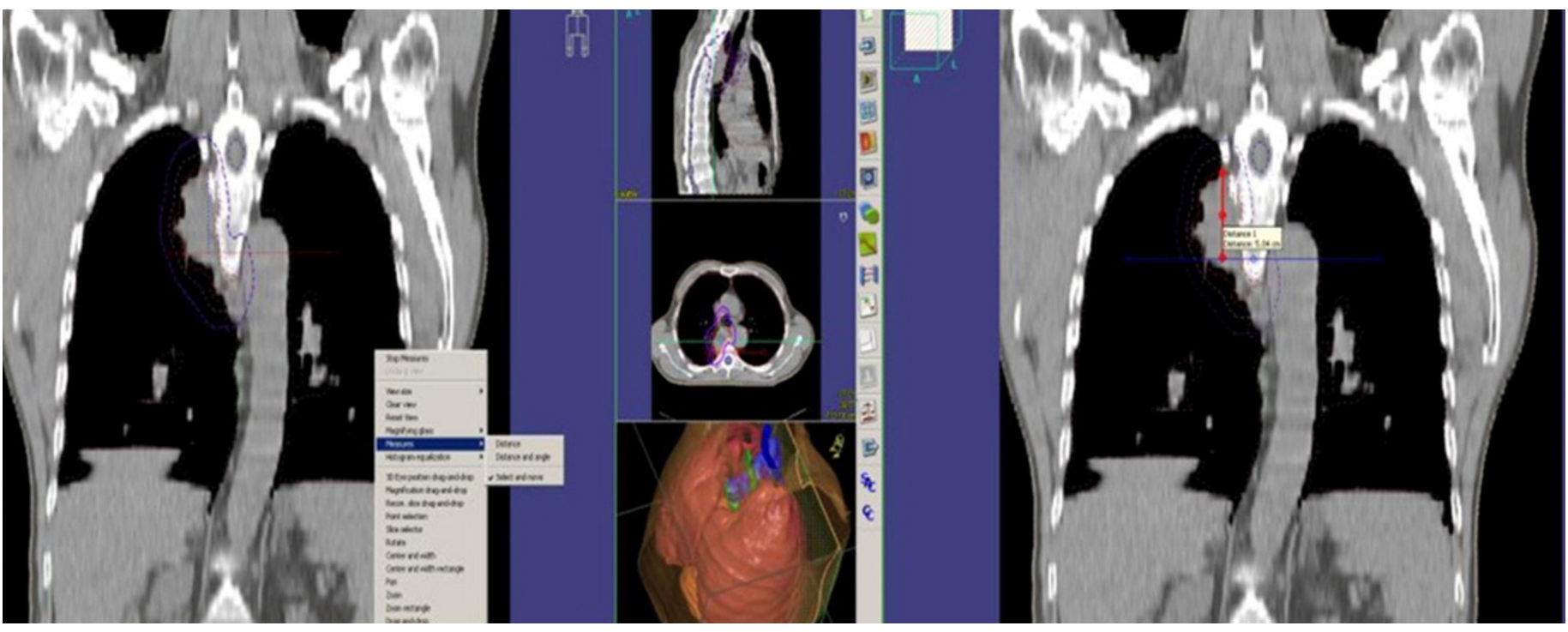

Figure $1 \mathbf{a}$, b. Horizontal reference line passing through the reference point (a). The CC distance between the upper most limit of the tumor in the cranial direction and the reference line (b). 
While the reference point in Plathow's study was the intersection point of midline passing through vertebrae and the anterior wall of the vertebral body at the T6/T7 intervertebral disk space, it was the intersection point of midline passing through vertebrae and anterior wall of the vertebral body at the most lower border of T5 vertebra in our study. Horizontal reference line passing through the reference point was plotted (Figure 1a).

The uppermost limit of the tumor in the cranial direction was determined and CC distance to the reference line (CC_BT) was measured (Figure 1b).

In the dynamic MRI images, coronal cross section images of the cases were used for determination of the reference line passing below T5 vertebral body. In dynamic MRI images, T5 vertebra could be identified in 9 of 12 patients. The lower border of the identified T5 vertebrae was determined as the reference line by the software program ISite PACS. In the evaluation of the inspiratory and expiratory phases, the position of the diaphragm dome in coronal cross sections was used (Figure 2).

In a manner similar to the measurement on coronal images of the CT-simulator, the uppermost cranial border of the tumor was determined in the sections showing peak of inspiration and expiration, and the $\mathrm{CC}$ distances to the reference line were measured $\left(\mathrm{CC}_{\text {ins }} \mathrm{MRI}, \mathrm{CC}_{\text {exp }} \mathrm{MRI}\right)$ (Figure 3).

$\mathrm{CC}_{\text {ins }} \mathrm{MRI}$, and $\mathrm{CC}_{\text {exp }} \mathrm{MRI}$ values were negative in cases whose uppermost cranial tumor border were located at the cranial side of the reference line, while positive if located at the caudal side of the reference line. The mean value of $C C_{\text {ins }} M R I$, and $C C_{\exp } M R I$ were calculated as mean CC MR (CC $\left.C_{\text {mean }} M R\right)$.

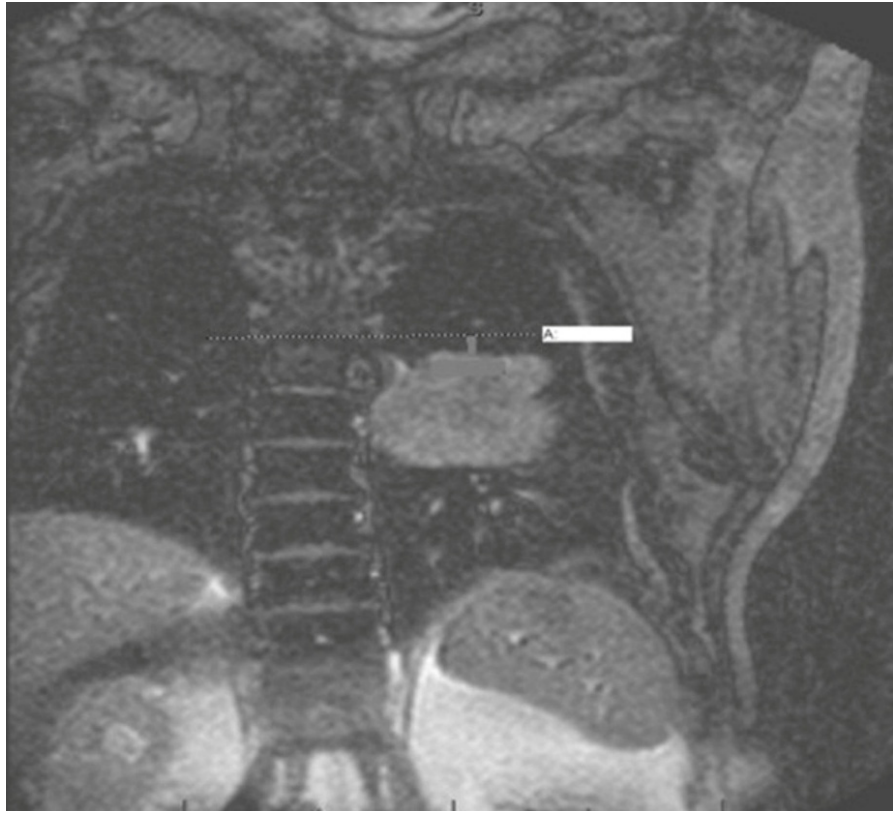

Figure 2. Determination of the reference line in dynamic MRI images.

The secondary aim of the study was to determine the maximum tumor motion in all axes in expirium and inspirium using dynamic MRI. First, the line passing through the center of the tumor on axial images was displayed in coronal images. In coronal cross sections, two lines tangent to the uppermost limit of the tumor (detected in the peak of inspiration and expiration) were drawn and copied to all cross sections. The distance between these two lines was measured (CC-MRI). In axial cross sections, two lines tangent to the to the lateral margin of the tumor detected in the peak of inspiration and expiration (determined by means

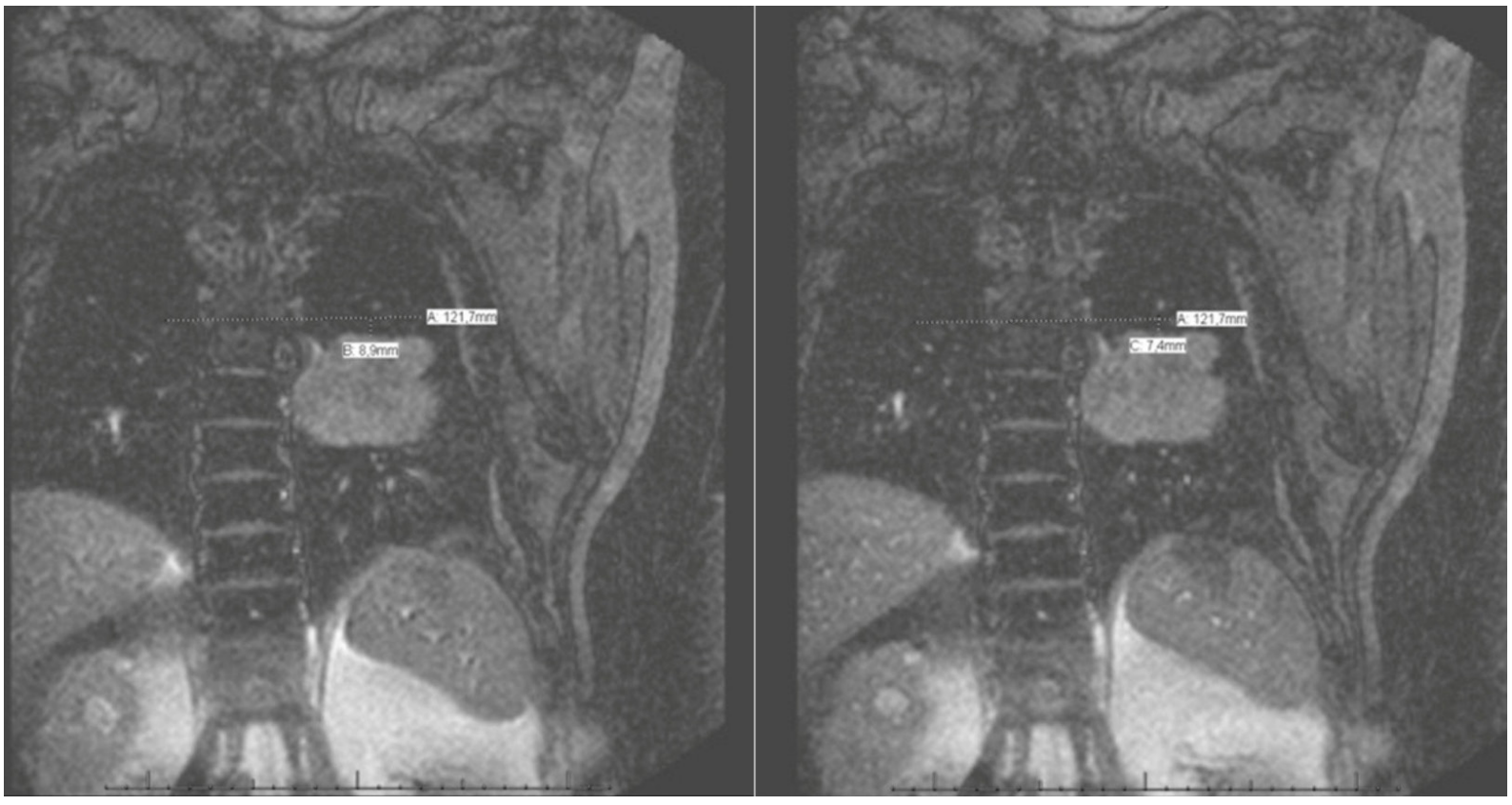

Figure $\mathbf{3} \mathbf{a}, \mathbf{b}$. The CC distances to the reference line in the peak of inspiration (a). The CC distances to the reference line in the peak of expiration (b). 


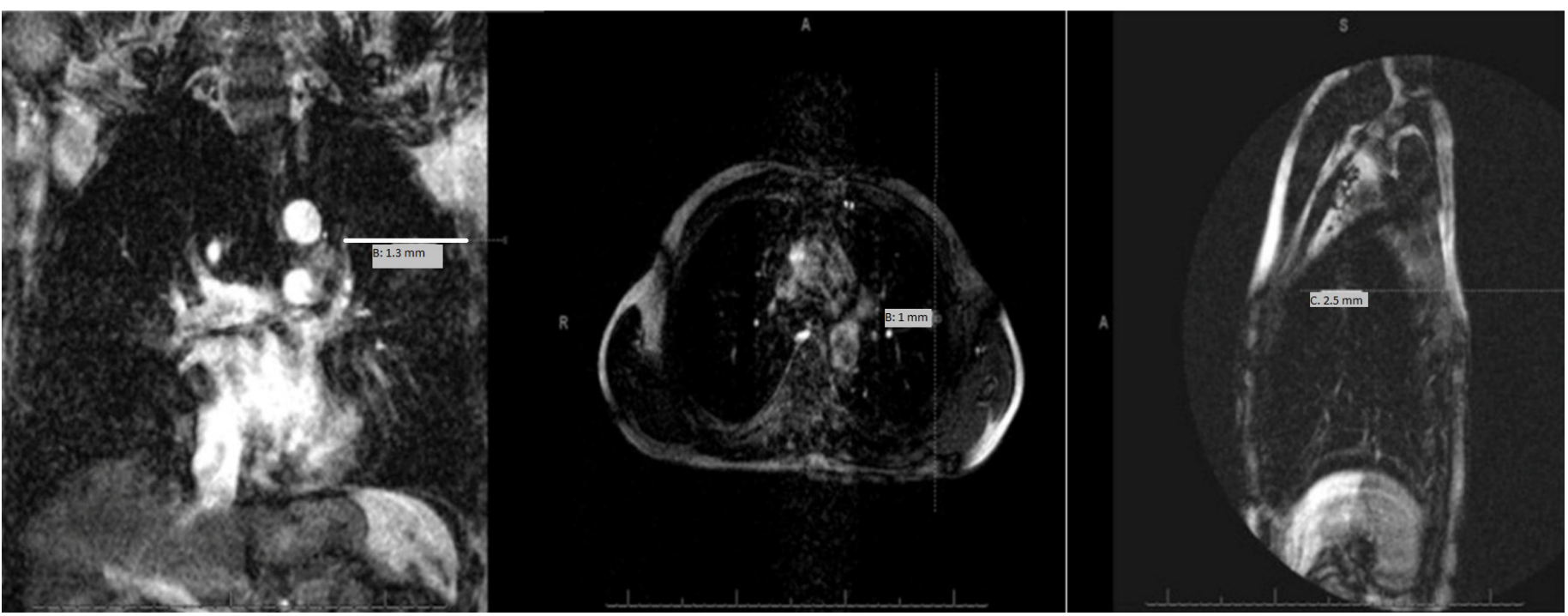

Figure 4. The measurement of maximum tumor motion in craniocaudal, mediolateral, anteroposterior directions in coronal, axial and sagittal dynamic MRI images.

of chest wall movement) were drawn and copied to all sections. The distance between these two lines was measured (ML_MRI). In sagittal cross sections, two lines tangent to the anterior border of the tumor detected in the peak of inspiration and expiration (determined by means of diaphragm movement) were drawn and copied to all sections. The distance between these two lines was measured (AP_MRI) (Figure 4).

\section{Statistical analysis}

Since the study population was less than 30 , it was decided that a non-parametric test should be used. Besides, the Wilcoxon signed rank test was preferred since two related data would need to be compared. $\mathrm{P}$ values were considered to be less than 0.05 for statistical significance.

Spearman's correlation test was used to evaluate the correlation between tumor location determined with CT-simulator and the average tumor location determined with dynamic MRI.

\section{RESULTS}

In the study, images acquired with dynamic MR technique displayed diaphragm and chest wall movements.

\section{Three dimensional motion of tumor}

The magnitudes of maximum tumor motion (CC-MRI, MLMRI, AP-MRI) under FB conditions were detected in three (craniocaudal, mediolateral, anteroposterior) axes in dynamic MRI (Table 1 and 2).

Average values of CC-MRI, ML-MRI and AP-MRI were $0.298 \pm$ $0.065,0.221 \pm 0.054$, and $0.202 \pm 0.034 \mathrm{~cm}$, respectively.

\section{Determination of CC distances in CT and dynamic MR images}

For all patients, CC distances between the upper limit of the GTV and the reference line were determined in $\mathrm{CT}$-simulator and dynamic MRI images (Table 3).
Table 1. The Mean Values and the Ranges of Maximum Tumor Motion (CC-MRI, ML-MRI, AP-MRI) Under Free Breathing Condition in Three (Craniocaudal, Mediolateral, Anteroposterior) Axes in Dynamic MRI

\begin{tabular}{lccc} 
& $\mathrm{CC}_{\mathrm{MR}}(\mathrm{cm})$ & $\mathrm{ML}_{\mathrm{MR}}(\mathrm{cm})$ & $\mathrm{AP}_{\mathrm{MR}}(\mathrm{cm})$ \\
\hline Mean $\pm \mathrm{SD}$ & $0.298 \pm 0.065$ & $0.221 \pm 0.054$ & $0.202 \pm 0.034$ \\
Minimum & 0.11 & 0.10 & 0.10 \\
Maximum & 0.73 & 0.70 & 0.50 \\
\hline
\end{tabular}

$\mathrm{CC}_{\mathrm{MR}}$, tumor motion measured with MRI in the craniocaudal axis. $\mathrm{ML}_{\mathrm{MR}}$, tumor motion measured with MRI in the mediolateral axis. $A P_{M R^{\prime}}$ tumor motion measured with $M R I$ in the anteroposterior axis.

Table 2. Maximum Tumor Motion Values Under Free Breathing Condition in Three (Craniocaudal, Mediolateral, Anteroposterior) Axes in Dynamic MRI

\begin{tabular}{cccc} 
Patient no & $\mathrm{CC}_{\mathrm{MR}}(\mathrm{cm})$ & $\mathrm{AP}_{\mathrm{MR}}(\mathrm{cm})$ & $\mathrm{ML}_{\mathrm{MR}}(\mathrm{cm})$ \\
1 & 0.15 & $0.35^{*}$ & 0.15 \\
2 & 0.15 & 0.10 & 0.10 \\
3 & 0.15 & 0.15 & 0.10 \\
4 & 0.11 & 0.15 & 0.10 \\
5 & 0.33 & 0.20 & 0.15 \\
6 & 0.65 & 0.10 & $0.70^{*}$ \\
7 & 0.73 & 0.22 & 0.15 \\
8 & 0.14 & 0.15 & 0.35 \\
9 & 0.12 & 0.25 & 0.10 \\
10 & 0.55 & 0.10 & 0.15 \\
11 & 0.15 & 0.50 & $0.45^{*}$ \\
12 & 0.35 & 0.15 & 0.15 \\
\hline
\end{tabular}

$\mathrm{CC}_{\mathrm{MR}}$, tumor motion measured with MRI in the craniocaudal axis. $\mathrm{ML}_{\mathrm{MR}}{ }^{\prime}$ tumor motion measured with MRI in the mediolateral axis. $\mathrm{AP}_{\mathrm{MR}}$, tumor motion measured with $\mathrm{MRI}$ in the anteroposterior axis. ${ }^{*}$ cardiac motion. 


\begin{tabular}{|c|c|c|c|c|c|c|c|c|c|}
\hline Patient no & 1 & 2 & 3 & 4 & 5 & 6 & 7 & 8 & 9 \\
\hline $\mathrm{CC}_{\exp }-\mathrm{MR}(\mathrm{cm})$ & -0.74 & 5.05 & 7.57 & 0.78 & -1.15 & -3.77 & -9.68 & -4.16 & -1.38 \\
\hline $\mathrm{CC}_{\text {mean }}-\mathrm{MR}(\mathrm{cm})$ & -0.82 & 5.13 & 7.5 & 0.84 & -1.32 & -3.45 & -10.1 & -4.23 & -1.44 \\
\hline$\left(\mathrm{CC}_{\text {mean }}-\mathrm{MR}\right)-(\mathrm{CC}-\mathrm{CT})(\mathrm{cm})$ & 0.01 & 0.04 & 0.02 & 0.06 & 0.14 & 0.06 & 0.01 & 0.05 & 0.01 \\
\hline
\end{tabular}

$\mathrm{CC}_{\text {ins }}-\mathrm{MR}$, craniocaudal distance measured in inspirium phase of MRI.

$C C_{\text {ins }}-M R$, craniocaudal distance measured in expirium phase of MRI.

$\mathrm{CC}_{\text {man }}-\mathrm{MR}$, the mean value of craniocaudal distance in MRI.

CC-CT, craniocaudal distance in the CT-simulator.

The range of $\mathrm{CC}_{\text {ins }}-\mathrm{MRI}$ was $0.89-10.5 \mathrm{~cm}$ in the caudal direction and $0.89-7.42 \mathrm{~cm}$ in the cranial direction. The range of $\mathrm{CC}_{\exp }-\mathrm{MRI}$ was $0.74-9.68 \mathrm{~cm}$ in the caudal direction and $0.78-7.57 \mathrm{~cm}$ in the cranial direction. The range of $\mathrm{CC}_{\text {mean }}-\mathrm{MRI}$ was $0.82-10.1 \mathrm{~cm}$ in the caudal direction, and $0.84-7.5 \mathrm{~cm}$ in the cranial direction. The range of CC-CT was found as $0.81-10.12 \mathrm{~cm}$ in the caudal direction and $0.78-7.48 \mathrm{~cm}$ in the cranial direction.

In Figure 3, the distribution of the CC distances between the upper limit of GTV and the reference line with respect to patients is shown.

Table 4 shows the differences between CC distances from the upper border of the GTV to the reference line and CC mean values in dynamic MRI.

The range of $\left|\left(C C_{\text {ins }}-M R\right)-\left(C C_{\text {mean }}-M R\right)\right|$ was $0.06-0.40 \mathrm{~cm}$. and the range of $\left|\left(C C_{\exp }-M R\right)-\left(C C_{\text {mean }}-M R\right)\right|$ was $0.06-0.33 \mathrm{~cm}$ (Table 4).

The mean difference between $\mathrm{CC}_{\text {mean }}-\mathrm{MRI}$ and CC-CT was 0.044 $(0.01-0.14) \mathrm{cm}$. In comparison of CC-CT and $\mathrm{CC}_{\text {mean }}-\mathrm{MRI}$ values by Wilcoxon signed rank test, no statistically significant difference was detected between CT-simulator and dynamic MRI $(p=0.172)$. When Spearman's correlation test was performed for CC-CT and $\mathrm{CC}_{\text {mean }}-\mathrm{MRI}$ values, it revealed a high level of correlation between these two imaging methods (Correlation coefficient: 0.983; $\mathrm{p}<0.0001)$.

\section{DISCUSSION}

In 3DCRT planning of LC, the most important factor affecting PTV is respiration-induced tumor motion. When PTV safety margins are not given in correct directions and amounts, normal tissues may be exposed to overdose and/or inadequate dose may result within GTV. Consequently, serious side effects due to RT (e.g. radiation pneumonitis, pulmonary fibrosis) and/or local-regional recurrence may occur.

There are many methods to reduce uncertainty concerning respiration-derived tumor motion. Fluoroscopy, $A B C$, "gating"
Table 4. The Differences between the "CC Distance in the Peak of Inspirium" and "the Mean CC Distance" and the Differences between the "CC Distance in the Peak of Expirium" and "the Mean CC Distance" in Dynamic MRI

\begin{tabular}{ccc}
\hline Patient no & $\begin{array}{c}\left|\left(\mathrm{CC}_{\text {ins }}-\mathrm{MR}\right)-\left(\mathrm{CC}_{\text {mean }}-\mathrm{MR}\right)\right| \\
(\mathrm{cm})\end{array}$ & $\begin{array}{c}\left|\left(\mathrm{CC}_{\text {exp }}-\mathrm{MR}\right)-\left(\mathrm{CC}_{\text {mean }}-\mathrm{MR}\right)\right| \\
(\mathrm{cm})\end{array}$ \\
\hline 1 & 0.07 & 0.08 \\
2 & 0.07 & 0.08 \\
3 & 0.08 & 0.07 \\
4 & 0.05 & 0.06 \\
5 & 0.16 & 0.17 \\
6 & 0.33 & 0.32 \\
7 & 0.40 & 0.33 \\
8 & 0.07 & 0.07 \\
9 & 0.06 & 0.06 \\
\hline
\end{tabular}

$\mathrm{CC}_{\text {ins }}-\mathrm{MR}$, craniocaudal distance measured in inspirium phase of MRI. $\mathrm{CC}_{\text {exp }}-\mathrm{MR}$, craniocaudal distance measured in expirium phase of MRI. $\mathrm{CC}_{\text {mean }}^{\text {exp }}-\mathrm{MR}$, the mean value of craniocaudal distance in MRI.

with markers placed on the skin, "tumor tracking" with implanted markers inside the tumor, the use of CT imaging, and MR-guided radiotherapy are some of them.

In fluoroscopy method, lung tumor motion during breathing cycle is examined in conventional simulator. Some disadvantages of this method are recognized. Fluoroscopy is a method that can show $\mathrm{CC}$ tumor motion, but not $\mathrm{ML}$ and $\mathrm{AP}$ tumor motion because of the presence of mediastinum. In order to control if tumor moves outside the defined PTV during the course of treatment, it is indicated that fluoroscopy method should be considered individually for each patient. Another problem regarding this method is the difficulty in transferring data of tumor motion to the geometry of the planning CT (6).

In the studies with $A B C$, patients are asked to hold their breath at a certain level during RT. ABC is performed during CT-simulation as well as during each treatment session. However, since this method 
requires patient cooperation, it can only be used in patients with normal pulmonary function tests and in non-elderly patients having relatively good performance status (10-12).

Another strategy is the "gating" method which monitors skinmarkers placed on skin or physiological motion $(10,13,14)$. In this method, "gating" is performed primarily during the CT-simulation session. Thus, CT cross sections are taken in a respiratory phase during which lung tumor motion is minimal. On these cross sections PTV is formed adding smaller safety margins with respect to the conditions without "gating" and the treatment plan which implements the most appropriate dose distribution in PTV is selected. Then, "gating" is performed during each treatment session in the same respiratory phase as in CT-simulation. However, the disadvantage of this system is the fact that skin marker motion may not always be consistent with the depth of breathing $(10,15)$.

Another way of "tracking" respiratory-derived tumor motion is to place markers directly into the tumor, instead of the skin. In this method, after inserting gold marker (GM) implants into or nearby the tumor using bronchoscopy, the tumor is monitored real time with fluoroscopy system located in the linear accelerator room. However, inserting implants carries the risk of bleeding due to close proximity to blood vessels in central tumors; that's why the implants are preferred rather in peripheral tumors. Similarly, due to the difficulty in insertion, this method is disadvantageous for tumors located in the upper lobes or upper segments of the lung. Again, due to the fragility of the tumor tissue, it is difficult for implanted GMs to remain stable throughout RT course. Also, in patients with post-operative bronchial stenosis or deformation, problems are known to arise in inserting GMs. One of the most important limitations of this invasive method is that the success rate depends, to a great extent, on the experience of the person inserting the implants (10).

RT is applied under normal respiratory conditions, thus the most frequently performed procedure is to take CT scans under normal respiratory conditions. For the PTV to be drawn as accurately as possible, slow CT scanning is performed at the speed of 4 seconds/cross section which corresponds to the duration of entire respiratory cycle. Reproducibility of PTV has been shown by this method (16).

Besides, 3D assessment of respiration-induced tumor motion can be performed by using multidetector CT (17). In a study by Hof et al., after exerting pressure on the abdomen, three consecutive CT scans were taken during FB, deep inspiration and deep expiration at a speed of 0.75 seconds per scan (17). The disadvantage of this technique has been indicated to be dependency on the patient's compliance (9). In general, disadvantages of the CT techniques trying to manage tumor motion are mainly instantaneous changes in tumor motion due to irregularity in respiration and additional exposure of the patient to radiation.

Another modality in managing tumor motion is MR-linac which is an advanced technology that combines two technologies-an MRI scanner and a linear accelerator-in radiation therapy. MR- imaging provides better tumour visibility than cone beam CT (CBCT) offering anatomical information and soft tissue imaging. These advantages of MR-imaging during treatment play a key role in the precise treatment in radiation oncology without exposure of the patient to additional radiation. Due to patient and field setup uncertainties during radiation delivery, MR-guided radiation therapy offers more accurate tumor tracking than $C B C T$ so that treatment can be adjusted accordingly. Therefore, MR-guided radiation therapy is an option that could provide real time visualization for real adaptive radiation therapy.

In a study of Plathow et al. including 39 patients with solitary non-small cell LC, respiratory cycle was imaged using "trueFISP" sequence (3 sections/second) in dynamic MRI (9). Lung and tumor mobility during respiration were analyzed in these images. In detecting tumor mobility, measurements were performed in FB as well as deep inspiration and deep expiration. In that study, CC, AP and ML motion of tumor was measured in the coronal, sagittal, and transverse plane, respectively. The reference lines for finding the amount of CC, AP and ML motion of the tumor were T6/T7 intervertebral disc space, tangential line to the anterior border of vertebrae, and vertical midline of the vertebrae. At the end of the study, lower lobe tumors $>5 \mathrm{~cm}$ were found to move less in the CC direction compared to those of $<3 \mathrm{~cm}(1.8 \pm 1.0 \mathrm{~cm}$ vs $3.8 \pm 0.7$ $\mathrm{cm}(\mathrm{p}<0.01))(9)$.

In a study including 24 patients with stage II-IV LC, Kovacs et al. performed dynamic MRI examination in the axial, sagittal and coronal planes during FB (100 section/30 seconds) (18). In this study, the amount of tumor motion in $A P, C C$, and $M L$ directions were determined by a software program for upper and lower lobe lung tumors. The average amounts of tumor motion were determined as AP $0.11 \mathrm{~cm}, \mathrm{ML} 0.11 \mathrm{~cm}$, and CC $0.27 \mathrm{~cm}$. These measurements were recognized as PTV safety margins that should be considered in the 3DCRT of patients with LC (18).

Although these studies have determined PTV safety margins by using dynamic MRI, the correlation between CT-simulator and dynamic MRI images with regard to CC tumor location has not been examined. However, in our study, in addition to the magnitudes of the respiratory-induced tumor motion (RITM) in $C C, A P$ and $M L$ planes in dynamic MRI, the correlation of CC tumor location in the CT-simulator and average CC location in the dynamic MRI was also studied.

Patients breathe freely during RT, thus CT-simulator scan acquisition used for RT planning is also performed during FB. Deep inspiration and deep expiration are not commonly seen during both CT-simulation and RT. Therefore, unlike Plathow's study, deep inspiration and deep expiration dynamic MRI data were not used in our study.

Our tumor mobility data calculated in three axes is similar to Kovacs et al.'s data. On the other hand, the amount of tumor mobility was reported higher in Plathow et al.'s study. compared to Kovacs et al.'s and our study. This may be due to the fact that Plathow et al.'s study includes patients who had stage I non-small 


\section{The distribution of the CC distances between the upper limit of GTV and the reference line}

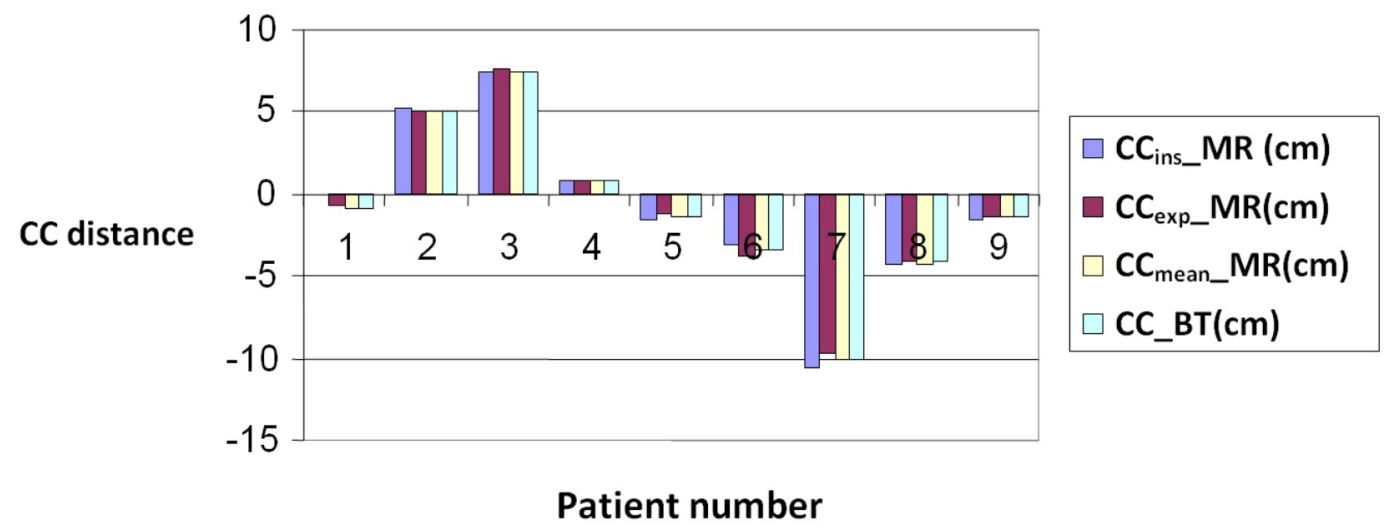

Figure 5. The CC distances between the upper most limit of GTV and the reference line (CT-simulator data and dynamic MRI data).

cell LC, $1 / 3$ of whom having tumors $<3 \mathrm{~cm}$, without any chest wall, mediastinum and vertebra invasion. On the other hand, stage IIIV patients with LC were included in our study similar to the study by Kovacs et al.

In our study, similar to the findings in Plathow et al.'s and Kovacs et al.' studies, the highest amount of RITM was observed in the CC axis. Due to the limited number of cases, it was impossible to analyze whether the RITM is influenced by patient's age, respiratory function tests, tumor size, and tumor location.

In our study, comparison of dynamic MRI and CT-simulator methods showed no significant difference in terms of CC location of tumor $(p=0.172)$. In addition, a high level of correlation was observed between the CC location of the tumor in CT-simulator and the average CC location of the tumor in dynamic MRI $(p<0.0001)$.

In most of our cases, CC-CT value was similar to the $\mathrm{CC}_{\text {mean }}-\mathrm{MRI}$ value, which indicates the actual average location of the tumor during respiration. As shown in Figure 5, CC-CT value was close to the $\mathrm{CC}_{\text {exp }}-\mathrm{MRI}$ value in case 4 and case 8, while close to the $\mathrm{CC}_{\text {ins }}$ MRI value in case 5 . Tumors of these patients were either small or located in the lower lobes. As shown in the study by Plathow et al., RITM was higher in cases with lower lobe or small sized tumors, suggesting that unlike dynamic MRI, CT-simulator scans may not show the average tumor location in these cases.

In the Radiation Oncology Clinic of our hospital, PTV margin, which is used in planning the appropriate beam design for tumor dose, is generally $1 \mathrm{~cm}$ in CC axis. In dynamic MRI images acquired in our study, the difference between CC distances from GTV upper border to the reference line in inspiration and expiration and the mean CC distance was maximum $0.4 \mathrm{~cm}$. However, PTV margin includes uncertainties not only due to respiratory motion but also due to set-up errors. In this sense, PTV margin given in CC axis in our clinic is considered to be adequate.

\section{CONCLUSION}

Dynamic MRI is a sensitive and well tolerated method enabling monitoring of tumor motion for 3DCRT treatment planning in LC. Our results demonstrate that respiratory induced tumor motion is seen at its maximum level in the $\mathrm{CC}$ axis.

In this study, comparison between dynamic MRI and CT-simulator methods did not show any significant difference in terms of CC location of the tumor, and these two methods displayed a high level of correlation. As a result of the evaluation of the CC tumor motion in dynamic MRI, PTV margin given in CC axis in our clinic is seen to be adequate. However, dynamic MRI is recommended particularly for the treatment planning of cases with small sized, lower-lobe and peripheral tumors since these tumors show higher mobility.

In the future, in order to detect tumor motion and project this accurately to treatment planning and treatment procedures, linear accelerators integrated with dynamic MRI-based techniques without additional radiation dose will be used, and this development may further improve the efficiency of RT. 
Informed Consent: Written informed consent was obtained from patient who participated in this study.

Peer-review: Externally peer-reviewed.

Author Contributions: Concept - SSN, CU, BA, AND; Design - SSN, NK, CU, BA, AND Supervision - SSN, AND; Resources - AY, AND; Materials - SSN, AND; Data Collection and/or Processing - SSN, AY, NK, CU, BA, AND; Analysis and/or Interpretation - SSN AY, AND; Literature Search - SSN, AY, CU, BA, AND; Writing Manuscript - SSN, AY, AND; Critical Review - SSN, AY, CU, BA, AND

Conflict of Interest: No conflict of interest was declared by the authors.

Financial Disclosure: The authors declared that this study has received no financial support.

\section{REFERENCES}

1. Heelan RT, Demas BE, Carevelli JF, et al. Superior sulcus tumors: CT and MR imaging. Radiology 1989;170:637-641. [CrossRef]

2. Pauls $S$, Breining $T$, Muche $R$, et al. The role of dynamic, contrastenhanced MRI in differentiating lung tumor subtypes. Clin Imaging 2011;35:259-265. [CrossRef]

3. Kramer H, Groen HJM. Current concepts in the mediastinal lymph node staging of nonsmall cell lung cancer. Ann Surg 2003;238:180188. [CrossRef]

4. Tavlayan E. The effect of CT and MRI image registration on target volume determination and dose distribution in the radiotherapy planning of brain tumors. Master of Science Thesis. Izmir; 2006. pp.1-6.

5. Shimizu S, Shirato H, Kagei $\mathrm{K}$, et al. Impact of respiratory movement on the computed tomographic images of small lung tumors in three-dimensional (3D) radiotherapy. Int J Radiat Oncol Biol Phys 2000;46:1127-1133. [CrossRef]

6. Stevens CW, Munden RF, Forster KM, et al. Respiratory-driven lung tumor motion in depend of tumor size, tumor location, and pulmonary function. Int J Radiat Oncol Biol Phys 2001;51:62-68. [CrossRef]

7. Gierada DS, Curtin J, Erickson SJ, Prost RW, Strandt JA, Goodman LR. Diaphragmatic motion: fast gradient-recalled-echo MR imaging in healthy subjects. Radiology 1995;194:879-884. [CrossRef]
8. Napadow VJ, Mai V, Bankier A, Gilbert RJ, Edelman R, Chen Q. Determination of regional pulmonary parenchymal strain during normal respiration using spin inversion tagged magnetization MRI. J Magn Reson Imaging 2001;13:467-474. [CrossRef]

9. Plathow C, Fink C, Ley S, et al. Measurement of tumor diameterdependent mobility of lung tumors by dynamic MRI. Radiother Oncol 2004;73:349-354. [CrossRef]

10. Harada T, Shirato H, Ogura S, et al. Real-time tumor-tracking radiation therapy for lung carcinoma by the aid of insertion of a gold marker using bronchofiberscopy. Cancer 2002;95:1720-1727. [CrossRef]

11. Wong JW, Sharpe MB, Jaffray DA, et al. The use of active breathing control $(A B C)$ to reduce margin for breathing motion. Int J Radiat Oncol Biol Phys 1999;44:911-919. [CrossRef]

12. Lohr F, Debus J, Frank C, et al. Noninvasive patient fixation for extracranial stereotactic radiotherapy. Int J Radiat Oncol Biol Phys 1999;45:521-527. [CrossRef]

13. Ohara K, Okumura T, Akisada M, et al. Irradiation synchronized with respiration gate. Int J Radiat Oncol Biol Phys 1989;17:853-857. [CrossRef]

14. Kubo HD, Hill BC. Respiration gated radiotherapy treatment: a technical study. Phys Med Biol 1996;41:83-91. [CrossRef]

15. Shimizu S, Shirato $H$, Aoyama $H$, et al. High-speed magnetic resonance imaging for four-dimensional treatment planning of conformal radiotherapy of moving body tumors. Int J Radiat Oncol Biol Phys 2000;48:471-474. [CrossRef]

16. Lagerwaard FJ, Van Sornsen de Koste JR, Nijssen-Visser MR, et al. Multiple slow CT scans for incorporating lung tumor mobility in radiotherapy planning. Int J Radiat Oncol Biol Phys 2001;51:932-937. [CrossRef]

17. Hof H, Herfarth KK, Münter M, Essig M, Wannenmacher M, Debus J. The use of the multislice $\mathrm{CT}$ for the determination of respiratory lung tumor movement in stereotactic single-dose irradiation. Strahlenther Onkol 2003;8:542-547. [CrossRef]

18. Kovacs A, Hadjiev J, Lakosi F, et al. Dynamic MR Based Analysis of Tumor Movement in Upper and Mid Lobe Localized Lung Cancer. Pathol Oncol Res 2009;15:269-277. [CrossRef] 